[2] Adorini L. (2005) Intervention in autoimmunity: the potential of vitamin D receptor agonists. Cell Immunol 233: 115-124

[3] Hewison M. (2012) Vitamin D and immune function: autocrine, paracrine or endocrine? Scand J Clin Lab Invest Suppl 243: 92-102

Disclosure of Interests: None declared

DOI: 10.1136/annrheumdis-2019-eular.3913

\begin{tabular}{|l|l|}
\hline AB0328 & SECONDARY SARCOPENIA IN RHEUMATOID \\
ARTHRITIS PATIENTS TREATED BY BIOLOGIC \\
DISEASE MODIFYING ANTI-RHEUMATIC DRUGS
\end{tabular}

Eriko Hasegawa ${ }^{1,2}$, Satoshi Ito $^{2}$, Yoichi Kurosawa ${ }^{1,2}$, Daisuke Kobayashi ${ }^{1}$ Asami Abe $^{2}$, Hiroshi Otani ${ }^{2}$, Kiyoshi Nakazono ${ }^{2}$, Akira Murasawa ${ }^{2}$, Ichiei Narita ${ }^{1}$ Hajime Ishikawa ${ }^{2} .{ }^{1}$ Niigata University Graduate School of Medical and Dental Sciences, Division of Clinical Nephrology and Rheumatology, Niigata City, Japan; ${ }^{2}$ Niigata Rheumatic Center, Department of Rheumatology, Shibata City, Japan

Background: Sarcopenia is characterized by a loss of muscle mass and strength, which leads to a reduced physical ability, poor quality of life $(\mathrm{QoL})$, frailty and mortality. Rheumatoid arthritis (RA) is considered a cause of secondary sarcopenia.

Objectives: To clarify the effectiveness of biologic disease-modifying antirheumatic drugs (bDMARDs) on sarcopenia, including the physical ability, body composition and nutritional status.

Methods: This was a prospective cohort study including consecutive 41 patients (11 men, 30 women, 63.6 \pm 16.1 years old) with RA who started bDMARDs for the first time at Niigata Rheumatic Center. The diagnosis of secondary sarcopenia was made according to the diagnostic algorithm of the Asian Working Group for Sarcopenia (AWGS), excluding the criteria about older age. We observed the disease activity of RA, physical ability, body composition, nutritional status and QoL at baseline and 6 months. The disease activity was assessed by the disease activity score28 joint count erythrocyte sedimentation rate (DAS28-ESR) and clinical disease activity index (CDAl). The physical activity was determined using the health assessment questionnaire (HAQ), 10-m walking test (10MWT) and timed up and go test (TUG). The nutritional status was determined based on the controlling nutrition status (CONUT) score and prognostic nutritional index (PNI). The overall QoL was measured by European quality of life scale- 5 dimensions (EQ-5D).

Results: Among 41 patients who started bDMARDs, 19 were classified as having sarcopenia, and 7 were classified as having pre-sarcopenia. The bDMARD was certolizumab pegol in 10 patients, adalimumab in 7 , abatacept in 7 , golimumab in 6 , tocilizumab in 5 , infliximab in 3 and etanercept in 3 . The DAS28-ESR $(4.7 \pm 1.3$ vs. $2.6 \pm 1.3, p<0.001)$ and CDAl $(18.6 \pm 9.4$ vs. $7.2 \pm 7.3, p<0.001)$ decreased significantly after 6 months of bDMARDs therapy. The physical activity was significantly improved after 6 months of bDMARDs: HAQ $(1.1 \pm 0.9$ vs. $0.7 \pm 0.9, p<0.001), 10 M W T$ $(1.5 \pm 0.7$ vs. $1.6 \pm 0.6 \mathrm{~m} / \mathrm{s}, \mathrm{p}=0.046)$ and TUG $(10.0 \pm 5.0$ vs. $9.5 \pm 6.2 \mathrm{~s}$, $p=0.024)$. Regarding the nutritional status, the CONUT score $(3.8 \pm 0.5$ vs. $1.1 \pm 1.2, \quad \mathrm{p}<0.001)$ and $\mathrm{PNI}(44.9 \pm 6.4$ vs. $49.7 \pm 4.1, \mathrm{p}<0.001)$ were significantly improved after 6 months of bDMARDs. The EQ5D was also improved after 6 months of bDMARDs $(0.64 \pm 0.15$ vs. $0.71 \pm 0.20$, $\mathrm{p}=0.010$ ). The body composition analysis showed a significant increase in the body weight $(54.3 \pm 13.2$ vs. $55.4 \pm 14.4 \mathrm{~kg}, \mathrm{p}=0.006)$ and fat mass $(16.3 \pm 7.3$ vs. $17.4 \pm 7.8 \mathrm{~kg}, p<0.001)$ after 6 months of bDMARDs but no significant increase in the appendicular skeletal muscle mass $(14.7 \pm 4.3$ vs. $14.8 \pm 4.5, \mathrm{p}=0.111)$. The proportion of patients classified as having sarcopenia showed a decreasing trend after 6 months of bDMARDs therapy $(46.3 \%$ vs. $24.4 \%, \mathrm{p}=0.0637)$.

Conclusion: After 6 months of bDMARDs therapy, the physical ability, nutritional status and QoL were significantly ameliorated. While the muscle mass was not markedly increased, the proportion of patients with sarcopenia showed a decreasing trend. The administration of bDMARDs might be useful for preventing secondary sarcopenia in RA patients.

Disclosure of Interests: Eriko Hasegawa: None declared, Satoshi Ito Speakers bureau: Mitsubishi Tanabe Pharma Corporation., Yoichi Kurosawa: None declared, Daisuke Kobayashi: None declared, Asami Abe: None declared, Hiroshi Otani: None declared, Kiyoshi Nakazono: None declared, Akira Murasawa: None declared, Ichiei Narita: None declared, Hajime Ishikawa: None declared

DOI: 10.1136/annrheumdis-2019-eular.4572

\section{AB0329 CERVICAL SPINE INVOLVEMENT IN RHEUMATOID ARTHRITIS}

Zsofia Kardos ${ }^{1}$, Csaba Oláh ${ }^{1}$, László Kostyál ${ }^{1}$, Katalin Hodosi ${ }^{2}$, László Tamási ${ }^{1}$, Zoltán Szekanecz ${ }^{2}{ }^{1}$ Borsod County Hospital, Miskolc, Hungary, ${ }^{2}$ University of Debrecen, Debrecen, Hungary

Background: After the small peripheral joints, the cervical spine is the second most involved region in rheumatoid arthritis (RA). The most frequent radiological features are the atlantoaxial subluxation (AAS) which can be anterior, posterior or vertical. During the course of the disease, the affection of the cervical spine has no symptoms for a long time due to the adaptability of neurological structures. The onset of myelopathy can occur at any time. MRI assessment compared to functional cervical spine X-ray is more sensitive method to provide not only AAS but also soft tissue involvement such as periodontal synovitis or fibrous pannus and even more odontoid erosion. New data show that there is a decreasing prevalence of cervical involvement because of the biologics. Objectives: We assessed RA patients in permanent remission with MR imaging. RA patients have no cervical pain or any neurological symptoms. We wished to explore the cervical spine involvement: AAS, odontoid erosion or periodontal soft tissue thickening. We also wished to determine the affectation of cervical spine in RA patients receiving different treatment strategies.

Methods: Altogether $49 \mathrm{RA}$ female patients were included. Among them, 15 were MTX-treated, biologic-free, 34 patients received biologics (17 infliximab [IFX] and 17 tocilizumab [TCZ]) as first-line biologic treatment, in combination with MTX. There was no significant difference between the main characteristics of these subgroups. ESR, CRP and DAS28 were determined in all RA patients in every 3 months. We calculated sumESR, sumCRP and sumDAS28 indices from the past 3 years.

Results: We detected anterior AAS in one-quarter of RA patients (13 affected patients from the total 49$)(26,5 \%)$. There was no significant difference between the therapeutic subgroups. No posterior or vertical AAS occurred. Compared with patients without cervical involvement, the patients with AAS showed higher sumCRP and sumESR levels, higher sumDAS28 scores and more frequent seropositivity, but these differences were not significant. Soft tissue involvement of the cervical spine was detected in $33,3 \%$ of MTX-treated, in $35,3 \%$ of IFX-treated and in $5,9 \%$ of TCZ-treated RA patients. Eight RA patients had odontoid erosion $(16,3 \%), 3$ from the MTX, 2 from the IFX and 3 from the TCZ-treated subgroups. In relation to soft tissue involvement and odontoid erosion we did not find any correlation with age, disease duration, seropositivity, sumESR, sumCRP or sumDAS28 indices.

Conclusion: These findings suggest that the presence of cervical involvement in RA patients is an important and frequent phenomenon even in asymptomatic patients. Higher ACPA titer, high disease activity and erosive disease at baseline are predictors of atlantoaxial involvement. With the appropriate disease control with conventional or biologic treatment progression of cervical spine involvement can also be prevented.

\section{REFERENCES}

[1] The Craniovertebral Junction in Rheumatoid Arthritis: State of the Art. Ferrante A, Ciccia F, Giammalva GR, lacopino DG, Visocchi M, Macaluso F, Maugeri R. Acta Neurochir Suppl. 2019;125:79-86.

[2] Magnetic resonance imaging of the craniovertebral junction in early rheumatoid arthritis. Carotti M, Salaffi F, Di Carlo M, Sessa F, Giovagnoni A. Skeletal Radiol. 2018 Sep 11.

Disclosure of Interests: Zsofia Kardos: None declared, Csaba Oláh: None declared, László Kostyál: None declared, Katalin Hodosi: None declared, László Tamási: None declared, Zoltán Szekanecz Grant/research support from: Pfizer, UCB, Consultant for: Pfizer, Abbvie, Roche, Sanofi, Lilly, Novartis, Speakers bureau: Pfizer, Abbvie, Roche, Sanofi, Lilly, Novartis DOI: 10.1136/annrheumdis-2019-eular.2832 